\title{
Indocyanine Green-Loaded Nanocarriers as Contrast Agents for NIR Fluorescent Optical Imaging
}

\section{Yongping Chen ${ }^{1 *, \#, ~ G u i h a i ~ C h e n 2 *, \#, ~ Y o n g x i ~ Z h a o ~}{ }^{3}$ and Wei Wang ${ }^{4}$}

${ }^{1}$ Department of Biomedical Engineering, Johns Hopkins University, 720 Rutland Avenue, Baltimore, Maryland 21205, USA

${ }^{2}$ Department of Neurology, the First Affiliated Hospital of Anhui Medical University, Hefei 230022, Anhui Province, PR China

${ }^{3}$ Key Laboratory of Biomedical Information Engineering of Education Ministry, School of Life Science and Technology, Xi'an Jiaotong University, Xi'an, Shaanxi 710049,

PR China

${ }^{4}$ Department of Urology, the Second People's Hospital of Hefei, Hefei 23001, Anhui, PR China

\#Both the authors has contributed equally

Optical imaging approaches offer the potential for non-invasive diagnosis and real-time, high-resolution, in vivo disease monitoring. Usually using UV to near-infrared (NIR) light, optical imaging uses wavelength-dependent interactions (scattering, absorption and fluorescence) in tissues to yield unique contrasts. $650-900 \mathrm{~nm}$ wavelength NIR optical fluorescence imaging has become a particularly attractive technology for disease diagnosis, treatment monitoring, and drug screening due to its low absorption, and significant autofluorescence imaging depth in vivo [1-3]. However, the lack of significant endogenous fluorescence contrast limits the diagnosis ability of this technology. Therefore to increase the signal-to-noise ratio and improve imaging resolution, the development of exogenous NIR optical contrast agents is a necessity [4-6].

Currently the only NIR fluorescent contrast agent approved by the United States Food and Drug Administration (FDA) for direct administration in medical diagnostics is Indocyanine Green (ICG) $[7,8]$. ICG is a water-soluble, amphiphilic tricarbocyanine dye with the adsorption and emission maxima around 780 and $810 \mathrm{~nm}$, respectively [9]. Due to its low toxicity ( $\mathrm{LD}_{50}$ of $50-80 \mathrm{mg} / \mathrm{kg}$ for animal subjects) [10] and capacity to absorb and emit in the NIR spectral range, ICG is used clinically as a contrast agent for visualizing blood and clearance, studying liver function, and guiding biopsies [11-13]. Despite its many advantages, ICG is still limited by several drawbacks: i) it aggregates easily into amphiphilic molecules in aqueous solutions to induce self quenching and low quantum yields [14]; ii) when administered in molecular form, ICG is rapidly cleared from the body with a short half-life about 2-4 min [15-17]; iii) it often binds to proteins leading to rapid agglomeration [11]; iv) it undergoes oxidation and dimerization, resulting in decreased absorption/emission and variability in the maximum absorption wavelength $[18,19]$; v) it is instable in aqueous solutions and prone to photobleaching under light exposure [16,20]; vi) ICG lacks target moieties for molecular imaging. To address these intrinsic drawbacks of ICG for in vivo imaging, a potential approach is to encapsulate ICG into nanocarriers that provide increased stability, protection from nonspecific plasma protein binding, prolonged circulation times and potential targeting. This editorial will focus on some of recent advances in the design of NIR contrast agents based on nanovector encapsulation of ICG.

There are many of reports that ICG encapsulators, such as (poly (lactic-coglycolic acid)(PLGA) nanoparticles (diameter $\sim 360 \mathrm{~nm}$ ) and silica-polymer composite microcapsules (diameter $\sim 0.6$ to $2 \mu \mathrm{m}$ ) improve the molecular instability of ICG and prolong its plasma halflife $[21,22]$. However, both of these nanoparticles are limited in size for in vivo tumor imaging depending on their EPR effects. Recently, several publications have reported promising results using smaller nanoparticles to encapsulate ICG for in vivo imaging. For example, Zheng et al. [23] developed ICG encapsulated PLGA-lipid nanoparticles conjugated with folic acid (FA) and demonsstrated their use as NIR contrast agents for tumor diagnosis and targeted imaging [23]. Altinoglu et al. also synthesized biodegradable calcium phosphosilicate nanoparticles (CPNPs) and demonstrated that small size $(16 \mathrm{~nm})$ ICG-encapsulating CPNPs have significantly better contrast agent optical properties than free fluorophores for tumor imaging [24]. Other inorganic delivery systems using silica nanoparticles have been developed to encapsulate ICG, and the ICG-SiO ${ }_{2}$ nanoparticles have the potential to be used as contrast agents for optical NIR imaging as well [25].

Among these nanocarriers, micelles are one of the successful types of drug delivery systems for in vivo applications due to their small size (approximately 10-100 $\mathrm{nm}$ ), which reduce clearance by the reticuloendothelial system (RES) and allow for an enhanced EPR effect $[26,27]$. Therefore, the encapsulation and stabilization of ICG dye as a contrast agent in micellar systems is of particular interest. For example, Pluronic F-127 (PF-127) polymeric micelles are approved by the FDA and have been successfully demonstrated to encapsulate and stabilize ICG as an NIR contrast agent for optical imaging $[28,29]$. Encapsulation of ICG within various micellar systems was also investigated by Kirchherr and co-workers, and they found many micellar systems improved the optical properties and stability of the ICG [30]. More interestingly, Zheng et al. [23] have recently reported a dual-functional ICG-PL-PEG agent with several unique features for optical imaging and photo-therapy [31]. This may emerge as a new strategy for combining tumor treatment and diagnosis together, using nanovectors with ICG.

In summary, this editorial discussed recent developments in nanocarrier ICG contrast agents for NIR optical imaging. Here just some of the areas are collected in terms of subjects and interests but it is hoped that every reader will find something of interest to them.

*Corresponding authors: Yongping Chen, Department of Biomedical Engineering Johns Hopkins University, 720 Rutland Avenue, Baltimore, Maryland 21205, USA, E-mail: yongpc@jhu.edu

Guihai Chen, Department of Neurology, the First Affiliated Hospital of Anhui Medical University, Hefei 230022, Anhui Province, PR China, E-mail: chenguihai1964@163.com

Received October 18, 2012; Accepted October 18, 2012; Published October 21, 2012

Citation: Chen Y, Chen G, Zhao Y, Wang W (2012) Indocyanine Green-Loaded Nanocarriers as Contrast Agents for NIR Fluorescent Optical Imaging. J Nanomed Nanotechol 3:e122. doi:10.4172/2157-7439.1000e122

Copyright: ( 2012 Chen Y, et al. This is an open-access article distributed under the terms of the Creative Commons Attribution License, which permits unrestricted use, distribution, and reproduction in any medium, provided the original author and source are credited. 
Citation: Chen Y, Chen G, Zhao Y, Wang W (2012) Indocyanine Green-Loaded Nanocarriers as Contrast Agents for NIR Fluorescent Optical Imaging. J Nanomed Nanotechol 3:e122. doi:10.4172/2157-7439.1000e122

\section{References}

1. Ke S, Wen X, Gurfinkel M, Charnsangavej C, Wallace S, et al. (2003) Nearinfrared optical imaging of epidermal growth factor receptor in breast cancer xenografts. Cancer Res 63:7870-7875.

2. Hilderbrand SA, Weissleder R (2010) Near-infrared fluorescence: application to in vivo molecular imaging. Curr Opin Chem Biol 14: 71-79.

3. Frangioni JV (2003) In vivo near-infrared fluorescence imaging. Curr Opin Chem Biol 7: 626-634.

4. Sevick-Muraca EM, Houston JP, Gurfinkel M (2002) Fluorescence-enhanced near infrared diagnostic imaging with contrast agents. Curr Opin Chem Biol 6 : $642-650$

5. Hawrysz DJ, Sevick-Muraca EM (2000) Developments toward diagnostic breas cancer imaging using near-infrared optical measurements and fluorescent contrast agents. Neoplasia 2: 388-417.

6. Hellebust A, Richards-Kortum R (2012) Advances in molecular imaging targeted optical contrast agents for cancer diagnostics. Nanomedicine-Uk 7 : 429-445.

7. Ballou B, Ernst Lauren A, Waggoner Alan S (2005) Fluorescence imaging of tumors in vivo. Curr Med Chem 12: 795-805

8. Rao J, Dragulescu-Andrasi A, Yao H (2007) Fluorescence imaging in vivo: recent advances. Curr Opin Biotechnol 18:17-25.

9. Saxena V, Sadoqi M, Shao J (2004) Indocyanine green-loaded biodegradable nanoparticles: preparation, physicochemical characterization and in vitro release. Int J Pharm 278: 293-301.

10. Taichamn GC, Hendry PJ, Wilbert JK (1987) The Use of Cardio-Green for Intraoperative Vizualization of the Coronary Circulation: Evaluation of Myocardial Toxicity. Tex Heart I J 14: 133-138.

11. Paumgartner G, Probst $P$, Kraines R, Leevy CM (1970) Kinetics of indocyanine green removal from the blood. Ann N Y Acad Sci 170:134-47.

12. Maarek JM, Holschneider DP, Rubinstein EH (2007) Fluorescence dilution technique for measurement of cardiac output and circulating blood volume in healthy human subjects. Anesthesiology 106:491-498.

13. Motomura K, Inaji H, Komoike Y, Kasugai I Noguchi S, Koyama, et al. (1999) Node Biopsy Guided by Indocyanine Green Dye in Breast Cancer Patients. Jpn J Clin Oncol 29: 604-607

14. Cardillo JA, Jorge R, Costa RA, Nunes SM, Lavinsky D, et al. (2008) Experimental selective choriocapillaris photothrombosis using a modified indocyanine green formulation. Br J Ophthalmol 92:276-280.

15. Yoneya S, Saito T, Komatsu Y, Koyama I, Takahashi K, et al. (1998) Binding properties of indocyanine green in human blood. Invest Ophthalmol Visual Sci 39: $1286-1290$.
16. Desmettre T, Devoisselle JM, Mordon S (2000) Fluorescence properties and metabolic features of indocyanine green (ICG) as related to angiography. Surv Ophthalmol 45: 15-27.

17. Mordon S, Devoisselle JM, Soulie-Begu S, Desmettre T (1998) Indocyanine green: physicochemical factors affecting its fluorescence in vivo. Microvasc Res 55: 146-152.

18. Zhang Y, Wang M (2000) The Luminescent Properties and Photo-Decay of Sulfosalicylic Acid Doped ORMOSILs. Mater Lett 42: 86-91.

19. Saxena V, Sadoqi M, Shao J (2003) Degradation Kinetics of Indocyanine Green in Aqueous Solution. J Pharm Sci 92: 2090-2097.

20. Landsman MLJ, Kwant G, Mook GA, Zijlstra WG (1976) Light-Absorbing Properties, Stability, and Spectral Stabilization of Indocyanine Green. J App Physiol 40: 575-583.

21. Saxena V, Sadoqi M, Shao J (2004) Enhanced photo-stability, thermal-stability and aqueous-stability of indocyanine green in polymeric nanoparticulate systems. J Photochem Photobiol B 74: 29-38.

22. Yaseen MA, Yu J, Wong MS, Anvari Bn (2007) Stability assessment of indocyanine green within dextran-coated mesocapsules by absorbance spectroscopy. J Biomed Opt 12: 064031.

23. Zheng CF, Zheng MB, Gong P, Jia AG, Zhang PF, et al. (2012) Indocyanine green-loaded biodegradable tumor targeting nanoprobes for in vitro and in vivo imaging. Biomaterials 33: 5603-5609

24. Altinoglu El, Russin TJ, Kaiser JM, Barth BM, Eklund PC, et al. (2008) NearInfrared Emitting Fluorophore-Doped Calcium Phosphate Nanoparticles for In Vivo Imaging of Human Breast Cancer. ACS Nano 2: 2075-2084.

25. Liu H, Farrell S, Uhrich K (2000) Drug release characteristics of unimolecula polymeric micelles. J Controlled Release 68: 167-174.

26. Lavasanifar A, Samuel J, Kwon GS (2002) Poly (ethylene oxide)-block-poly (L-amino acid) micelles for drug delivery. Adv Drug Delivery Rev 54: 169-190.

27. Chen YP, Li XD (2011) Near-Infrared Fluorescent Nanocapsules with Reversible Response to Thermal/pH Modulation for Optical Imaging. Biomacromolecules 12: $4367-4372$

28. Kim TH, Chen Y, Mount CW, Gombotz WR, Li XD, et al. (2010) Evaluation of Temperature-Sensitive, Indocyanine Green-Encapsulating Micelles for Noninvasive Near-Infrared Tumor Imaging. Pharm Res 27: 1900-1913.

29. Kirchherr AK, Briel A, Mader K (2009) Stabilization of indocyanine green by encapsulation within micellar systems. Mol Pharmaceutics 6: 480-491.

30. Zheng XH, Xing D, Zhou FF, Wu BY, Chen ,et al. (2011) Indocyanine GreenContaining Nanostructure as Near Infrared Dual-Functional Targeting Probes for Optical Imaging and Photothermal Therapy. Mol Pharmaceutics 447-456.

31. Zheng XH, Zhou FF, Wu BY, Chen WR, Xing D (2012) Enhanced Tumo Treatment Using Biofunctional Indocyanine Green-Containing Nanostructure by Intratumoral or Intravenous Injection. Mol Pharmaceutics 9: 514-522. 